\title{
The significance of macroautophagy in health and disease
}

\author{
C. Tukaj \\ Department of Electron Microscopy, Medical University of Gdansk, Gdansk, Poland
}

[Received 31 December 2012; Accepted 18 January 2013]

\begin{abstract}
During the past decade there has been a substantial increase in macroautophagy (herein simply referred to as autophagy) research due to a growing understanding of this process, coupled with improved new techniques for its detection. Autophagy (auto - self, phagy - eating) is defined as a fundamental lysosomal catabolic pathway responsible for degrading long-lived proteins, protein aggregates, oxidised lipids, damaged organelles, and even microbial invaders. Although autophagy occurs at basal levels in normal conditions, many different forms of metabolic stress, including starvation, hypoxia, high temperature, high culture density, hormones, and growth factor deprivation can dramatically stimulate an autophagic response. Autophagy plays a critical role in maintaining cellular homeostasis and genomic integrity and therefore has been implicated in many physiological activities such development, differentiation, and tissue remodelling. Consequently, defects in autophagy have been linked to various human diseases such as neurodegenerative and muscle disorders, cancers, cardiac failure, and inflammatory disorders. This mini-review summarises current knowledge in a field of mammalian autophagy and considers the significance of autophagy in human physiology and pathology. (Folia Morphol 2013; 72, 2: 87-93)
\end{abstract}

Key words: autophagosome, autophagic response, cytoprotection, pathology

\section{INTRODUCTION}

The autophagic process was first described by Clark in 1957 using electron microscopy, but the term "autophagy" was introduced for the first time in 1963 by Christian de Duve, who was awarded the Nobel Prize for his work on lysosomes. Autophagy (auto - self, phagy - eating) is defined as an evolutionarily conserved and strictly regulated lysosomal pathway that degrades cytoplasmic material and organelles and even microbial invaders $[22,36,37,39]$. The other major degrading system, which is present only in mammals, is the ubiquitin-proteasome system (UPS) [44]. The UPS degrades soluble short-lived proteins, which must be tagged by ubiquitin to be recognised by the proteasome. The process of autophagy has different mechanisms and routes for the elimination of cytoplasmic components, and therefore it can be more precisely subdivided in 3 types: macroautophagy, microautophagy, and chaperone-mediated autophagy [54]. Micro- and macroautophagy are both highly evolutionary conserved from yeast to humans, while chaperone-mediated autophagy is a selective form of autophagy that has only been observed in mammalian cells $[37,50]$. Macroautophagy represents the most extensively studied form of autophagy and involves the prominent formation of double-membrane vesicles termed autophagosomes, which surround and randomly

Address for correspondence: C. Tukaj, PhD, Department of Electron Microscopy, Medical University of Gdansk, ul. Dębinki 1, 80-210 Gdańsk, Poland, tel: +48583491501, e-mail: ctukaj@gumed.edu.pl 


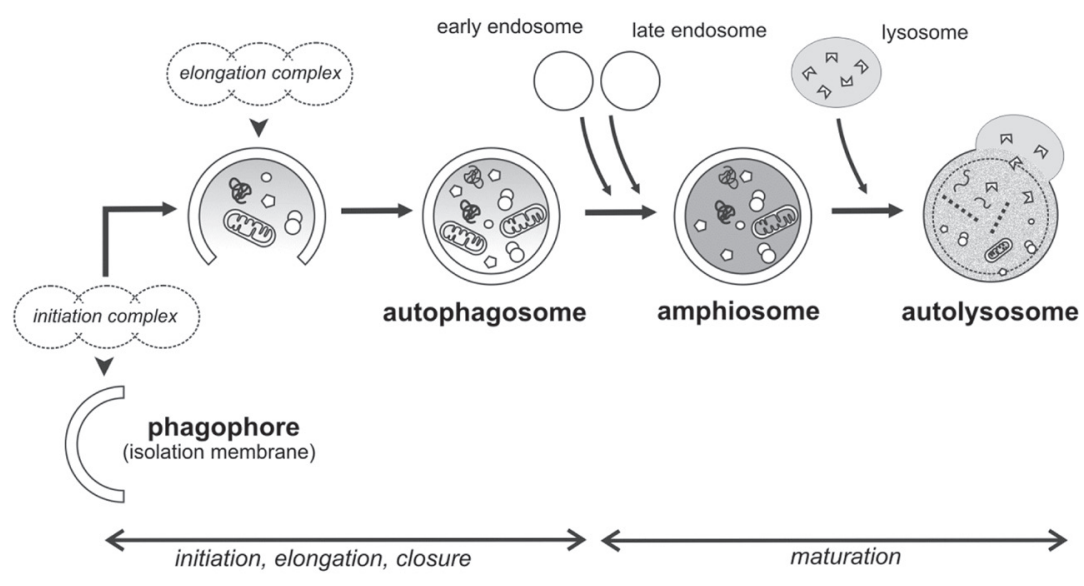

Figure 1. Schematic diagram of autophagic progression in mammalian cells. Following induction of autophagy, cytoplasmic components become sequestered by a flat membrane sheet known as the isolation membrane or phagophore. Finally, the expanding membrane sac fully encloses the object destined for elimination to form a double-membrane vesicle, the autophagosome. Fusion between the autophagosome and lysosome, often referred to as autophagic flux or maturation, generates an autolysosome (autophagolysosome). Intra-autophagosomal contents as well as the inner autophagosomal membrane are degraded by the lysosomal hydrolases. In the final step of autophagy, macromolecules generated from degraded cargo are transported back to the cytosol for reuse.

sequester cytoplasmic constituents prior to fusion with lysosomes [7, 22, 37, 48, 54]. In general, autophagy is considered to be a non-selective pro-survival cellular response; however, several forms of autophagy that display selectivity, e.g. mitophagy (degrades mitochondria), reticulophagy (degrades endoplasmic reticulum), xenophagy (degrades microorganisms), and aggrephagy (degrades aggregated proteins), have been recognised [18, 21]. Autophagy is extensively studied due to the identification of many autophagy-related genes (ATG) coupled with improved methods for monitoring this process $[23,30$, 43]. Although substantial progress has been achieved in elucidating the autophagic machinery, the mechanism of formation of autophagosome is still unclear. Several different assays are available to monitor autophagy, but using a combination of them is recommended. The most traditional method used to detect autophagosomes is electron microscopy; therefore, conventional morphometry is currently the standard for assessing autophagy, both in tissues and in cultured cells. Nevertheless, the electron microscopy method is limited in its potential, and thus new methods for identifying and quantifying and also for modulating autophagic activity was applied $[19,23,38,55]$. Fluorescence microscopy is a powerful method for the detection of molecules involved in the autophagosome biogenesis. To date, LC3 - a microtubule-associated protein 1 light chain 3 - is the only well-characterised protein that is specifically localised to autophagic structures throughout the process from phagophore to lysosomal degradation [55]. The number of autophagosomes usually correlates well with the amount of LC3-II punctate structures, and therefore it can usually be visualised using fluorescence microscopy and analysed with image analysis software. The LC3-II expression can be also quantitatively and sensitively monitored by immunoblot analysis or flow cytometry. Although LC3-II is a promising autophagosomal marker, it is necessary to carefully interpret the results because increased LC3-II levels can be associated with either enhanced autophagosome synthesis or reduced autophagosome turnover. Because autophagy is a dynamic and continuous process, the autophagy flux is necessary to differentiate whether the accumulated autophagosomes are due to increased production or reduced degradation. One of the principal methods to measure autophagic flux is the monitoring of LC3-II turnover, which is based on the observation of differences in LC3-II levels in the presence and absence of lysosome inhibitors, such as chloroquine [19, 55].

\section{THE AUTOPHAGOSOMAL PATHWAY}

Autophagy delivers cytoplasmic constituents to lysosomal degradation via the formation of an autophagosome $[7,8,20,30,43]$. Morphological trials of autophagy consist of several sequential stages (Fig. 1): initiation, nucleation, elongation, closure, maturation, and finally degradation and export of materials to the cytoplasm.

\section{Initiation}

In the initial step, a cup-shaped double membrane structure, known as the "isolation membrane", is formed (Fig. 1). This unique perivacuolar structure, termed the phagophore, has been proposed as the 
precursor of autophagosome - a double-membrane vesicle containing cytoplasmic constituents $[2,31,37$, $43,48,57]$. The exact origin of the isolation membrane is still controversial, but probably there is not a single source. It seems likely that the ribosome-free region of the rough endoplasmic reticulum, mitochondrial outer membrane, or Golgi compartments are the sources for the phagophore [13, 22].

\section{Elongation and closure}

Phagophore elongates and completely captures the sequestered cargo to form the double-membrane autophagosome, or "autophagic vacuole". Sequestration can be either nonspecific, involving the engulfment of bulk cytoplasm, or selective, targeting specific cargoes such as organelles or invasive microbes $[2,7,22]$. Autophagosomes form randomly in the cytoplasm and the size of typical autophagosomal double-membrane vacuoles ranges from 300 to $900 \mathrm{~nm}$. This step is a simple sequestration, and no degradation occurs.

\section{Maturation and degradation}

After formation, the completed autophagosome undergoes a stepwise maturation process including its conversion into a degradative organelle, termed autolysosome, by fusion with late endosomal and lysosomal organelles. Some recent studies have revealed the importance of cytoskeletal elements, such as actin microfilaments and microtubules, in specific aspects of autophagy [7, 24]. Autophagosomes are trafficked along microtubules in a dynein-dependent manner to lysosomes, which are clustered around the microtubule-organising centre (MTOC; located near the nucleus). There, autophagosome docks with the lysosome, and the outer membrane of the autophagosome successively fuses with the membrane of the lysosome. The inner membrane is finally consumed together with the cargo by resident hydrolases. In addition, the autophagosomes can first fuse with other vesicles including endosomes and multivesicular bodies to form hybrid organelles called amphisomes that later fuse with lysosomes, where the entrapped cytosolic contents are degraded [57]. It is currently not clear whether amphisome formation is a prerequisite for autophagosome-lysosome fusion. An autophagosome that has fused with a lysosome subsequently matures to become single-membrane degradative organelle autolysosome often called "autophagolysosome" [36, 40, 41, 54]. Acidification and acquisition of hydrolytic enzymes, including proteases, lipases, nucleases, and glycosidases, enable the autolysosome to degrade sequestered intra-autophagosomal components [30, 36]. The breakdown products are then transferred back into the cytoplasm where they can either be recycled or further catabolised. Whereas the mobility of autophagic vacuoles is dependent on microtubules, fusion seems to be independent of cytoskeletal elements [24]. The definition of the vacuoles named above is based on their function, not on their morphology. Therefore, it is not easy to distinguish autophagosomes, amphisomes, and autolysosomes by electron microscopy. Although some methods to monitor autophagy flux have been presented, the degradation step is rather difficult to measure [38]. In particular, distinction of autolysosomes from other cellular membranous compartments is often more difficult. The maturation of autophagosomes is a relatively quick process, and under starvation conditions all of the described events occur within 10-15 $\min [23,54]$.

\section{REGULATION OF AUTOPHAGIC ACTIVITY}

In mammals, the formation of autophagosomes appears to be a highly coordinated, multistep process controlled by a specific set of genes described by a common name, ATG. Genetic screening in yeast originally identified more than 30 different ATG genes, and many of these have mammalian homologs $[22,39,48,50]$. In the early stages of autophagosome formation, the nucleation of autophagic vacuoles requires the activity of the class-III phosphoinositide 3-kinase (PI3K), which is part of a large macromolecular specific complex that contains the important protein Atg6 (also called beclin 1) [3]. In mammals, at least 3 types of III PI3-kinase complexes contribute to autophagy, and thus autophagosome formation is positively regulated by Rab7 and the UV-RAG-Vps34-beclin1 PI3-kinase complex, whereas it is negatively regulated by the Rubicon-UV-RAG-Vsp34-beclin1 complex [50,61]. While PI3 generated by Beclin-1 containing complexes marks sites of autophagosome generation, 2 ubiquitin-like systems are required for elongation of phagophore and the maturation of autophagosomes [40,54]. Atg5 and Atg12 are involved in the first of 2 ubiquitylation-like reactions that interact non-covalently with Atg16L (Atg16-like) to form a complex. The second ubiquitylation-like reaction involves the cleavage of microtubule-associated protein 1 light chain 3 (MAP1-LC3) also known as Atg8 or LC3 in mammals, and its subsequent conjugation to a phospholipid, phosphatidylethanolamine (PE), to form LC3-II in the autophagosomal membrane $[3,8]$. LC3-II is a unique protein that specifically associates with newly forming autophagosome membranes and not with other vesicular structures [36]. LC3-II is present on both the cytosolic and the intra-autophagosomal surfaces and therefore increa- 


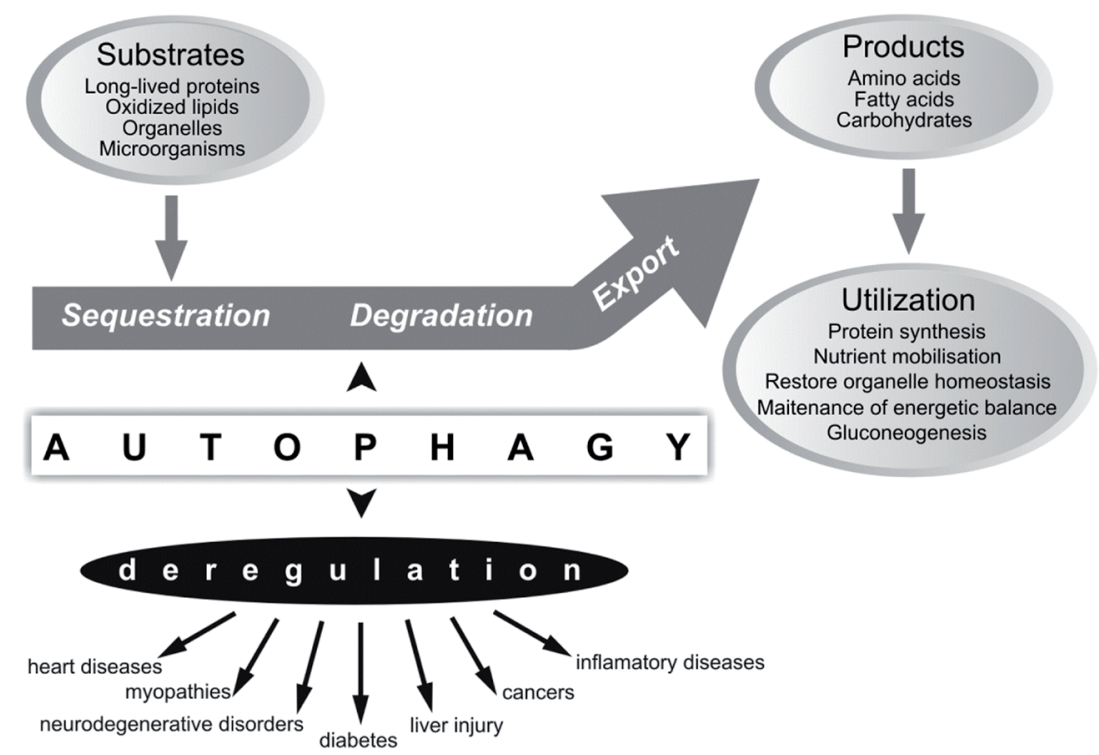

Figure 2. The role of autophagy in human physiology and disease (see text for details).

ses during autophagosome formation [40]. In mammals, the conversion of LC3 from LC3-I (free form) to LC3-II (PE-conjugated form) is regarded as a critical step in autophagosome formation [19]. Although Atg5-Atg12 is lost upon completion of autophagosome formation, LC3-II remains associated with autophagosomes even after fusion with lysosomes, after which LC3-II on the cytosolic face of autolysosomes can be delipidated and recycled (to LC3-I). Thus the level of LC3-II correlates with the autophagosome number, and this specific association makes it an excellent marker for monitoring autophagy [40]. Crosstalk between the 2 mentioned ubiquitylation-like systems has also been suggested. Moreover, autophagosome formation is tightly regulated by intracellular and extracellular amino acid concentrations and ATP levels via major inhibitory signalling pathways that include the mammalian target of rapamycin, mTOR kinase [59]. This evolutionarily conserved TOR kinase complex 1 is a sensor of nutrient status and plays an important role in the control of cell growth. More detailed information on molecular machinery that regulates and executes autophagy can be found in several recent reviews on these topics [22, 39, 51, 54].

\section{PHYSIOLOGICAL FUNCTIONS OF AUTOPHAGY}

Autophagy occurs constitutively at low basal levels in all eukaryotic cells where it operates as a cellular quality control mechanism responsible for the normal turnover of intracellular components and maintenance of the cellular energetic balance $[4,25,37,52]$. Under most cir- cumstances, autophagy constitutes a stress adaptation pathway that promotes cell survival, and thus has been implicated in many fundamental physiological activities such as development, differentiation, and tissue remodelling [2, 39]. The breakdown products - amino acids, fatty acids, nucleosides, and carbohydrates - generated by autophagy, released by lysosomal permeases, can be delivered systemically and exported to the cytosol for reuse (Fig. 2). Moreover, the amino acids liberated from autophagic degradation can be, together with the fatty acids, used by the tricarboxylic acid cycle to maintain cellular ATP production [3]. Autophagy can be rapidly upregulated by numerous stressors including, among others, nutrient starvation, hormone and growth factor deprivation, high temperature or hypoxia, or pharmacological agents (e.g. rapamycin) $[8,27,36]$. The best-known evolutionary conserved stress response in eukaryotes is stimulation of autophagy during a period of starvation, both in cultured cells and in intact organisms $[28,40,50]$. Recently, it was appreciated that macroautophagy not only contributes to the turnover of cytoplasmic constituents, but also targets intracellular pathogens for degradation during innate immunity [5]. This housekeeping function of autophagy includes the direct elimination of certain intracellular microbial pathogens via a process termed xenophagy. Eliminating many bacteria (group A Streptococcus, Staphylococcus aureus, Mycobacterium tuberculosis, Ricketssia coronii), parasites (Toxoplasma gondii), and viruses (herpes simplex virus type I), autophagy participates in the cell-autonomous antimicrobial defence system 
$[5,31]$. Moreover, autophagy plays critical functions in regulating adaptive immune responses, immunological tolerance, and the development and homeostasis of the immune system $[16,32,45]$. In nervous system, the stimulation of autophagy limits the accumulation of toxic products and protects neurons against degeneration $[15,50,53,57]$. In muscle the heart cells basal autophagy seems to have a special housekeeping role in the turnover of cytoplasmic constituents, including mitochondria, and is upregulated in response to stress [17]. The findings of LaRocca et al. [29] also suggest that autophagy preserves arterial endothelial function by reducing oxidative stress and inflammation and increasing nitric oxide bioavailability. It has also been suggested that autophagy participates in the degradation of mitochondria during reticulocyte maturation [12]. Lipid droplets from the liver are eliminated by their constitutive degradation in autolysosomes [48], but on the other hand, autophagy is required for the production of the large lipid droplets characteristic of the white adipose tissue. The anti-aging role of autophagy probably depends on its quality control function that limits the production of reactive oxygen species by the deposition of aggregation-prone proteins and damaged mitochondria $[1,4]$. Recent genetic analyses in various organisms have resulted in the identification of genes involved in controlling longevity $[6,10]$. Growing evidence has indicated both classical autophagy (macroautophagy) and chaperone-mediated autophagy decline with aging in rodents and probably in humans [49]. A cardinal feature of aging postmitotic cells is the accumulation of yellowish-brown, autofluorescent lipofuscin deposits [1].

A critical physiological role of autophagy appears to be cytoprotection, but this process is also associated with cell death through its intertwined relationships with apoptosis and necrosis [10, 11, 30, 42]. Some studies implicate that excessive degradation of cellular constituents contributes to a form of cell death, usually referred to as Type II programmed cell death or autophagic cell death (ACD), characterised by a large-scale sequestration of portions of the cytoplasm, giving the cell a characteristic vacuolated appearance. ACD also occurs in a caspase-independent manner, without key features of apoptotic morphology, such as chromatin condensation and nuclear fragmentation [27]. Nevertheless, autophagy and apoptosis share several common regulatory elements, which suggests a dual role of autophagy in cell survival and cell death $[6,11]$.

\section{AUTOPHAGY: RELATED PATHOLOGIES}

Alterations in autophagy have been linked to a wide range of pathological conditions including neurodegeneration, myopathy, liver disease, diabetes, and cancer [16, 30, 31, 36, 46, 51, 53, 58]. Especially in nonproliferative cells, such as neurones and myocytes where normal turnover of cytoplasmic contents may be critical, impairment of autophagy contribute to neurodegeneration or muscle atrophy $[42,56$, 59]. Examples demonstrate extended accumulation of polyglutamine-containing proteins that cause Huntington's disease, mutant forms of $\alpha$-synuclein that cause Parkinson's disease, and amyloid plaques consisting of $\beta$-amyloid $(\mathrm{A} \beta)$ peptides that cause Alzheimer's disease $[47,53,56,59]$. However, it is important to underline that the role of autophagy in protection against neurodegenerative diseases has been established in animal models but not yet in patients $[15,25,40]$. The other diseases associated with blockade of the autophagic pathway are muscular disorders, known as vacuolar myopathies [46]. Massive accumulation of imperfect autophagic or lysosomal vacuoles can be observed in patients suffering from Danon's disease, which has clinical characteristics including cardiomyopathy, myopathy, and variable mental retardation [50].

The contribution of autophagy to cancer development, especially in resistance to some anticancer treatments, is widely accepted. Although it is generally agreed that autophagy is low in cancer cells, increasing evidence supports both tumour promoting and suppressive functions of autophagy [11, 14]. It is hypothesised that autophagy maintains genomic integrity and thus would seem to be beneficial for cancer prevention during tumour initiation in certain circumstances $[33,35]$. The loss of the essential autophagy gene Beclin 1 (Atg6) has been observed in $40 \%$ to $75 \%$ of human breast, prostate, and ovarian cancers [58], suggesting that autophagy may play a role in preventing these tumours. Several preclinical and clinical studies indicate that pharmacological inhibition of mTOR, which leads to the induction of autophagy, provides a powerful therapeutic tool for the treatment of various malignancies [51,60]. In more advanced tumours during cancer progression, autophagy may, on the other hand, provide tumour cells with a survival strategy, thus suggesting a therapeutic use for autophagy down-regulation [reviewed in 31,36$]$. Additionally, understanding the relationship between autophagic and apoptotic pathways 
may provide novel therapeutic approaches to treating drug-resistant cancers.

The role of autophagy in immune response is not limited to the direct elimination of invading pathogens but also contributes to chronic inflammatory diseases and autoimmune diseases [16, 32, 45]. Perturbations in macroautophagy regulators have been reported in the Crohn's disease, an inflammatory bowel disorder affecting the small intestine [32]. Other potential links include systemic lupus erythematosus, inflammation-associated metabolic diseases such as obesity and diabetes, and inflammation associated with cystic fibrosis lung disease [34, 50]. Recent studies suggest that autophagy is necessary to maintain the structure, mass, and function of pancreatic $\beta$-cells under conditions such as oxidative stress, and that reduction in autophagic activity may contribute to a reduced ability to produce insulin [9]. It is hypothesised that the disturbance of autophagy function in the liver could lead to progressive accumulation of aggregation-prone $\alpha 1$-antitrypsin. Such perturbations in autophagy have been associated with chronic liver disease and hepatocellular carcinoma [26]. Undoubtedly, a better understanding of the complexity of autophagy and its regulation may allow the practical use of autophagy for therapeutic purposes.

\section{ACKNOWLEDGMENTS}

This study was supported by the Medical University of Gdansk within project ref. ST-59.

\section{REFERENCES}

1. Brunk UT, Terman A (2002) Lipofuscin: mechanisms of age-related accumulation and influence on cell functions. Free Radic Biol Med, 33: 611-619.

2. Cecconi F, Levine $B(2008)$ The role of autophagy in mammalian development: cell makeover rather than cell death. Dev Cell, 15: 344-357.

3. Chen Y, Klionsky DJ (2011) The regulation of autophagy: unanswered questions. J Cell Sci, 15: 161-170.

4. Cuervo AM (2004) Autophagy: in sickness and in health. Trends Cell Biol, 14: 70-77.

5. Deretic V, Levine B (2009) Autophagy, immunity, and microbial adaptations. Cell Host Microbe, 5: 527-549.

6. Eisenberg-Lerner A, Bialik S, Simon HU, Kimchi A (2009) Life and death partners: apoptosis, autophagy and the cross-talk between them. Cell Death Differ, 16: 966-975.

7. Eskelinen EL (2008) New insights into the mechanisms of macroautophagy in mammalian cells. Int Rev Cell Mol Biol, 266: 207-247.

8. Eskelinen EL, Saftig P (2009) Autophagy: a lysosomal degradation pathway with a central role in health and disease. Biochim Biophys Acta, 1793: 664-673.
9. Fujitani $\mathrm{Y}$, Ueno $\mathrm{T}$, Watada $\mathrm{H}$ (2010) Autophagy in health and disease. 4 . The role of pancreatic beta-cell autophagy in health and diabetes. Am J Physiol Cell Physiol, 299: 1-6.

10. Gozuacik D, Kimchi A (2007) Autophagy and cell death. Curr Top Dev Biol, 78: 217-245.

11. González-Polo RA, Boya P, Pauleau AL, Jalil A, Larochette N, Souquere S, Eskelinen EL, Pierron G, Saftig P, Kroemer G (2005) The apoptosis/autophagy paradox. Autophagic vacuolization before apoptotic death. J Cell Sci, 118: 3091-3102.

12. Griffiths RE, Kupzig S, Cogan N, Mankelow TJ, Betin VM, Trakarnsanga K, Massey EJ, Parsons SF, Anstee DJ, Lane JD (2012) The ins and outs of human reticulocyte maturation: autophagy and the endosome/exosome pathway. Autophagy, 8: 1150-1151.

13. Hailey DW, Rambold AS, Satpute-Krishnan P, Mitra K, Sougrat R, Kim PK, Lippincott-Schwartz J (2010) Mitochondria supply membranes for autophagosome biogenesis during starvation. Cell,141: 656-667.

14. Hanahan D, Weinberg RA (2011) Hallmarks of cancer: the next generation. Cell, 144: 646-674.

15. Hara T, Nakamura K, Matsui M, Yamamoto A, Nakahara $Y$, Suzuki-Migishima R, Yokoyama M, Mishima K, Saito I, Okano H, Mizushima N (2006) Suppression of basal autophagy in neural cells causes neurodegenerative disease in mice. Nature, 441: 885-889.

16. Heath RJ, Xavier R (2009) Autophagy, immunity and human disease. Curr Opin Gastroenterol, 25: 512-520.

17. Hill JA (2011) Autophagy in cardiac plasticity and disease. Pediatr Cardiol, 32: 282-289.

18. Johansen T, Lamark T (2011) Selective autophagy mediated by autophagic adapter proteins. Autophagy, 7: 279-296.

19. Kabeya $Y$, Mizushima N, Ueno T, Yamamoto A, Kirisako T, Noda T, Kominami E, Ohsumi Y, Yoshimori T (2000) LC3, a mammalian homologue of yeast Apg8p, is localized in autophagosome membranes after processing. EMBO J, 19: $5720-5728$.

20. Kang R, Zeh HJ, Lotze MT, Zeh HJ 3rd (2011) The Beclin 1 network regulates autophagy and apoptosis. Cell Death Differ, 18: 571-580.

21. Kim I, Rodriguez-Enriquez S, Lemasters JJ (2007) Selective degradation of mitochondria by mitophagy. Arch Biochem Biophys, 462: 245-253.

22. Klionsky DJ (2007) Autophagy: from phenomenology to molecular understanding in less than a decade. Nat Rev Mol Cell Biol, 8: 931-937.

23. Klionsky DJ, Cuervo AM, Seglen PO (2007) Methods for monitoring autophagy from yeast to human Autophagy, 3: 181-206.

24. Kochl R, Hu XW, Chan EY, Tooze SA (2006) Microtubules facilitate autophagosome formation and fusion of autophagosomes with endosomes. Traffic, 7: 129-145.

25. Komatsu M, Waguri S, Chiba T, Murata S, Iwata J, Tanida I, Ueno T, Koike M, Uchiyama Y, Kominami E, Tanaka K (2006) Loss of autophagy in the central nervous system causes neurodegeneration in mice. Nature, 441: 880-884.

26. Kotsafti A, Farinati F, Cardin R, Cillo U, Nitti D, Bortolami M (2012) Autophagy and apoptosis-related genes in chronic liver disease and hepatocellular carcinoma. BMC Gastroenterol, 12: 118. 
27. Kroemer G, Levine B (2008) Autophagic cell death: the story of a misnomer. Nat Rev Mol Cell Biol, 9: 1004-1010.

28. Kuma A, Hatano M, Matsui M, Yamamoto A, Nakaya $H$, Yoshimori T, Ohsumi Y, Tokuhisa T, Mizushima N (2004) The role of autophagy during the early neonatal starvation period. Nature, 432: 1032-1036.

29. LaRocca TJ, Henson GD, Thorburn A, Sindler AL, Pierce GL, Seals DR (2012) Translational evidence that impaired autophagy contributes to arterial ageing. J Physiol, 590: 3305-3316.

30. Levine B, Klionsky DJ (2004) Development by self-digestion: molecular mechanisms and biological functions of autophagy. Dev Cell, 6: 463-477.

31. Levine B, Kroemer G (2008) Autophagy in the pathogenesis of disease. Cell, 132: 27-42.

32. Levine B, Mizushima N, Virgin HW (2011) Autophagy in immunity and inflammation. Nature, 469: 323-335.

33. Liang C, Feng P, Ku B, Dotan I, Canaani D, Oh, BH, Jung JU (2006) Autophagic and tumour suppressor activity of a novel Beclin 1-binding protein UVRAG. Nat Cell Biol, 8: 688-699.

34. Luciani A, Villella VR, Esposito S, Brunetti-Pierri N, Medina DL, Settembre C, Gavina M, Raia V, Ballabio A, Maiuri L (2011) Cystic fibrosis: a disorder with defective autophagy. Autophagy, 7: 104-106.

35. Mathew R, Karantza-Wadsworth V, White E (2007) Role of autophagy in cancer. Nat Rev Cancer, 7: 961-967.

36. Mizushima N (2005) The pleiotropic role of autophagy: from protein metabolism to bactericide. Cell Death Differ, 12: 1535-1541.

37. Mizushima N (2007) Autophagy; process and function. Genes Dev, 21: 2861-2873.

38. Mizushima N (2010) Methods in mammalian autophagy research. Cell, 140: 313-326.

39. Mizushima N, Levine B (2010) Autophagy in mammalian development and differentiation. Nat Cell Biol, 12: 823-830.

40. Mizushima N, Yamamoto A, Matsui M, Yoshimori T, Ohsumi $Y$ (2004) In vivo analysis of autophagy in response to nutrient starvation using transgenic mice expressing a fluorescent autophagosome marker. Mol Biol Cell, 15: 1101-1111.

41. Mizushima N, Yoshimori T, Ohsumi Y (2011) The role of Atg proteins in autophagosome formation. Annu Rev Cell Dev Biol, 27: 107-132.

42. Moreau K, Luo S, Rubinsztein DC (2010) Cytoprotective roles for autophagy. Curr Opin Cell Biol, 22: 206-211.

43. Nakatogawa H, Suzuki K, Kamada Y, Ohsumi Y (2009) Dynamics and diversity in autophagy mechanisms: lessons from yeast. Nat Rev Mol Cell Biol, 10: 458-467.

44. Nedelsky NB, Todd PK, Taylor JP (2008) Autophagy and the ubiquitin-proteasome system: collaborators in neuroprotection. Biochim Biophys Acta, 1782: 691-699.

45. Nedjic J, Aichinger M, Emmerich J, Mizushima N, Klein L (2008) Autophagy in thymic epithelium shapes the T-cell repertoire and is essential for tolerance. Nature, 455: 396-400.
46. Nishino I (2006) Autophagic vacuolar myopathy. Semin Pediatr Neurol 13: 90-95.

47. Nixon R, Cataldo A (2006) Lysosomal disease pathways: genes to neurodegeneration in Alzheimer's disease. J Alzheimers Dis, 9: 277-289.

48. Rabinowitz JD, White E (2010) Autophagy and metabolism. Science, 330: 1344-1348.

49. Rajawat YS, Hilioti Z, Bossis I (2009) Aging: central role for autophagy and the lysosomal degradative system. Ageing Res Rev, 8: 199-213.

50. Ravikumar B, Sarkar S, Davies JE, Futter M, Garcia-Arencibia M, Green-Thompson ZW, Jimenez-Sanchez M, Korolchuk VI, Lichtenberg M, Luo S, Massey DC, Menzies FM, Moreau K, Narayanan U, Renna M, Siddiqi FH, Underwood BR, Winslow AR, Rubinsztein DC (2010) Regulation of mammalian autophagy in physiology and pathophysiology. Physiol Rev, 90: 1383-1435.

51. Rubinsztein DC, Codogno P, Levine B (2012) Autophagy modulation as a potential therapeutic target for diverse diseases. Nat Rev Drug Discov, 11: 709-730.

52. Singh R, Cuervo AM (2011) Autophagy in the cellular energetic balance. Cell Metabolism, 13: 495-504.

53. Son JH, Shim JH, Kim KH, Ha JY, Han JY (2012) Neuronal autophagy and neurodegenerative diseases. Exp Mol Med, 44: 89-98.

54. Tanida I (2011) Autophagosome formation and molecular mechanism of autophagy. Antioxid Redox Signal, 14: 2201-2214.

55. Tanida I, Minematsu-Ikeguchi N, Ueno T, Kominami E (2005) Lysosomal turnover, but not a cellular level, of endogenous LC3 is a marker for autophagy. Autophagy, 1: 84-91.

56. Winslow AR, Chen CW, Corrochano S, Acevedo-Arozena A, Gordon DE, Peden AA, Lichtenberg M, Menzies FM, Ravikumar B, Imarisio S, Brown S, O'Kane CJ, Rubinsztein DC (2010) Alpha-Synuclein impairs macroautophagy: implications for Parkinson's disease. J Cell Biol, 190: 1023-1037.

57. Wong AS, Cheung ZH, Ip NY (2011) Molecular machinery of macroautophagy and its deregulation in diseases. Biochim Biophys Acta, 1812: 1490-1497.

58. Yang ZJ, Chee CE, Huang S, Sinicrope FA (2011) The role of autophagy in cancer: therapeutic implications. Mol Cancer Ther, 10: 1533-1541.

59. Yu WH, Cuervo AM, Kumar A, Peterhoff CM, Schmidt SD, Lee JH, Mohan PS, Mercken M, Farmery MR, Tjernberg LO, Jiang $Y$, Duff $K$, Uchiyama $Y$, Näslund J, Mathews PM, Cataldo AM, Nixon RA (2005) Macroautophagy: a novel Beta-amyloid peptide-generating pathway activated in Alzheimer's disease. J Cell Biol, 171: 87-98.

60. Yu L, McPhee CK, Zheng L, Mardones GA, Rong Y, Peng J, Mi N, Zhao Y, Liu Z, Wan F, Hailey DW, Oorschot V, Klumperman J, Baehrecke EH, Lenardo MJ (2010) Termination of autophagy and reformation of lysosomes regulated by mTOR. Nature, 465: 942-946.

61. Zhong Y, Wang QJ, Li X, Yan Y, Backer JM, Chait BT, Heintz N, Yue Z (2009) Distinct regulation of autophagic activity by Atg14L and Rubicon associated with Beclin 1-phosphatidylinositol-3-kinase complex. Nat Cell Biol, 11: 468-476. 